\title{
Characteristics of the Analytic Network Process, a Multi-Criteria Decision-Making Method
}

\author{
Nikola Kadoić ${ }^{1, *}$ \\ ${ }^{1}$ Faculty of Organization and Informatics in Varaždin, University of Zagreb \\ Pavlinska 2, 42000, Varaždin, Croatia \\ E-mail:〈nkadoic@foi.hr〉
}

\begin{abstract}
The Analytic Network Process (ANP) is one of the most complex multi-criteria decisionmaking methods. It was developed by Professor Thomas Saaty, who also created the Analytic Hierarchy Process (AHP). In the network, we model the dependencies and influences between decision-making elements. A network contains much more information on the decision-making problem than the hierarchy does. By applying the ANP, we, therefore, obtain more accurate results (the decision). However, AHP is much more often applied even when the decision-making problems contain influences and dependencies which were not considered in the AHP. The main research goal of this paper is to identify reasons (ANP characteristics) why ANP is not applied when the problems contain influences and dependencies. After describing the main characteristics, we focus on the three characteristics of the ANP, which are (1) the inseparability of the criteria and alternatives, (2) the influence of the goal node on the priorities in the decision-making problem and (3) the stochasticity of the supermatrix in the ANP method. All these three characteristics are theoretically analyzed in depth and demonstrated through examples. The paper concludes with proposals on how the ANP can be used with respect to these three characteristics.
\end{abstract}

Key words: analytic network process, ANP, stochasticity, supermatrix, reducibility

Received: August 8, 2018; accepted: October 18, 2018; available online: December 13, 2018

DOI: $10.17535 /$ crorr.2018.0018

\section{Introduction}

This paper is prepared within the scope of the Higher Decision project. The main goal of this project is to develop a framework for strategic decision making in higher education (HE) which will support the process of decision making from the problem identification phase to an evaluation of the strategy selected as a solution for the identified problem [5]. The base of the phase in the framework, called making strategic decisions, is an Analytic Network Process (ANP) method. The ANP is one of the most complex multi-criteria decision-making methods; but on the other hand, the ANP is a method that takes into account the most data about decision-making problem compared to other multi-criteria decision-making methods. By using the ANP, it is possible to model dependencies and feedbacks in the network structure of a problem [18]. Most of the other methods do not support this feature [15]. However, literature review analysis has suggested that the methods which do not support modelling dependencies and feedback are much more often used in the practice [11]. The focus of this paper is analysis of the ANP and its characteristics.

The paper consists of several sections. In Section 2, the ANP is briefly presented and demonstrated using example; in Section 3, several characteristics of the ANP are listed according

${ }^{*}$ Corresponding author. 

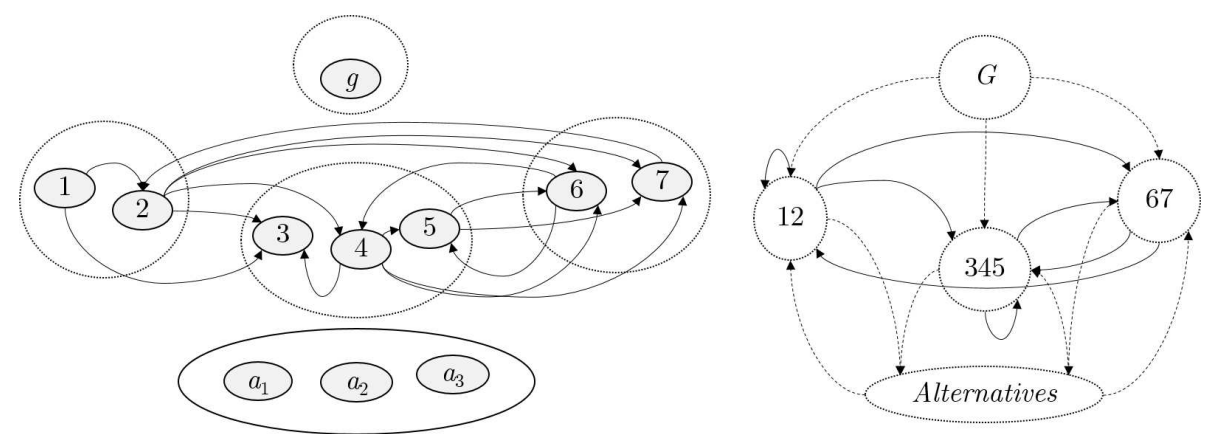

Figure 1: Network structure of the demo example; node (left) and cluster (right) levels.

to the literature review analysis and author's experience in using the ANP; an finally in Section 4, three characteristics of the ANP are analysed in detail. These characteristics are related to: (1) the inseparability of criteria and alternatives, (2) the influence of the goal node on the priorities in the decision-making problem and (3) the stochasticity of the supermatrix in the ANP method.

\section{The Analytic Network Process (ANP)}

The ANP is a generalization of the Analytic Hierarchy Process (AHP). Both the ANP and the ANP were founded by prof. Thomas Saaty [23]. In the AHP,a decision-making problem has a hierarchical structure; at the top is the decision-making goal, and at the second level are criteria which can be decomposed into subcriteria at a lower level. These subcriteria can be additionally decomposed into subsubcriteria and the decomposition could be in-depth, depending on the problem that is being analysed. The goal, criteria and subcriteria make a tree. The tree is then upgraded with the alternatives at the lowest level. The alternatives are connected to all (sub)(sub)criteria which are not decomposed into the lower level (the leaves of the tree) [20].

In the ANP, the hierarchal structure is upgraded to a network which allows interactions between elements of the hierarchy (e.g. a certain leaf could influence other leaves). The network in the ANP consists of clusters and elements [17, 22, 21]. To create a network, decision makers often combine the ANP with the Interpretative Structural Modelling (ISM) $[6,24,3]$ and/or the Decision Making Trial and Evaluation Laboratory (DEMATEL) [26, 7, 25, 27]. We will explain the ANP method using a demo example. The structure of this demo example is presented in Figure 1. The network structure of the demo example consists of three clusters of criteria (clusters 12, 234 and 67) and seven elements (criteria 1âĂŞ7). Additionally, the structure contains clusters and elements which are related to the decision-making goal and alternatives. The dependencies between the criteria are marked by arrows. The dependencies between node $g$ and the criteria, and between the alternatives and the criteria are not drawn in Figure 1 (node level) due to problems with opacity. At the cluster level, the dependencies are marked by dashed arrows. Additional information about dependencies, influences and structuring methods can be found in $[12,14,13]$. In general, the term dependency is the opposite to influence. From a mathematical point of view, the ANP is the only method which is proven to be eligible to analyze connections within a decision system including consistency check and eigenvector confirmation.

According to the ANP steps, after the problem is structured it is needed to calculate the weighted supermatrix. The procedure is as follows:

- The starting point is to make the empty supermatrix. The dimension of the matrix equals the number of nodes in the problem (11 in the demo example), 
- Then, pairwise comparisons at the node level must be done (the comparison procedure and Saaty's scale, which is a base of the comparison procedure, are explained in more detail in paper [13]):

- Comparisons of the criteria with respect to goals. Criteria from different clusters are compared separately (in the demo case, we will have three comparisons matrices since there are three clusters of criteria). The weights (comparisons results) have to be inserted into the first column of the supermatrix.

- Comparisons of criteria with respect to other criteria. Here, several comparisons have to be done: criteria 3 and 4 with respect to criterion 2 ; criteria 6 and 7 with respect to criterion 2 ; criteria 6 and 7 with respect to criterion 4 ; criteria 3 and 5 with respect to criterion 4 ; criteria 6 and 7 with respect to criterion 5 ; and criteria 4 and 6 with respect to criterion 6 . The results must be written in the columns of the unweighted supermatrix with respect to which pairwise comparisons are done. Additionally, when a criterion depends on only one other criterion from some cluster, weight 1 have to be inserted (e.g. criterion 1 depends on only criterion 3 from cluster 345 , so 1 will be inserted in unweighted supermatrix, column 1, row 3),

- Pairwise comparisons of the alternatives with respect to each criterion (the results must be inserted into the columns of the criteria and the rows of the alternatives), and pairwise comparisons of the criteria with respect to the alternatives (the results have to be inserted into the columns of the alternatives and the rows of the criteria). On all other positions of unweighted supermatrix we write 0 . The matrix has to be converted to weighted supermatrix now,

- Afterwards, the pairwise comparisons on the cluster level must be done:

- Comparing clusters 12, 345 and 67 with respect to the cluster $G$,

- Comparing clusters 12, 345 and 67 with respect to the cluster Alternatives,

- Comparing clusters 12, 345, 67 and the Alternatives with respect to 12,

- Comparing clusters 345, 67 and the Alternatives with respect to cluster 345,

- Comparing clusters 12, 345 and the Alternatives with respect to 67.

The weights obtained through pairwise comparisons prodecure on the cluster level multiply the related blocks of the unweighted supermatrix which then becomes a (stochastic) weighted supermatrix.

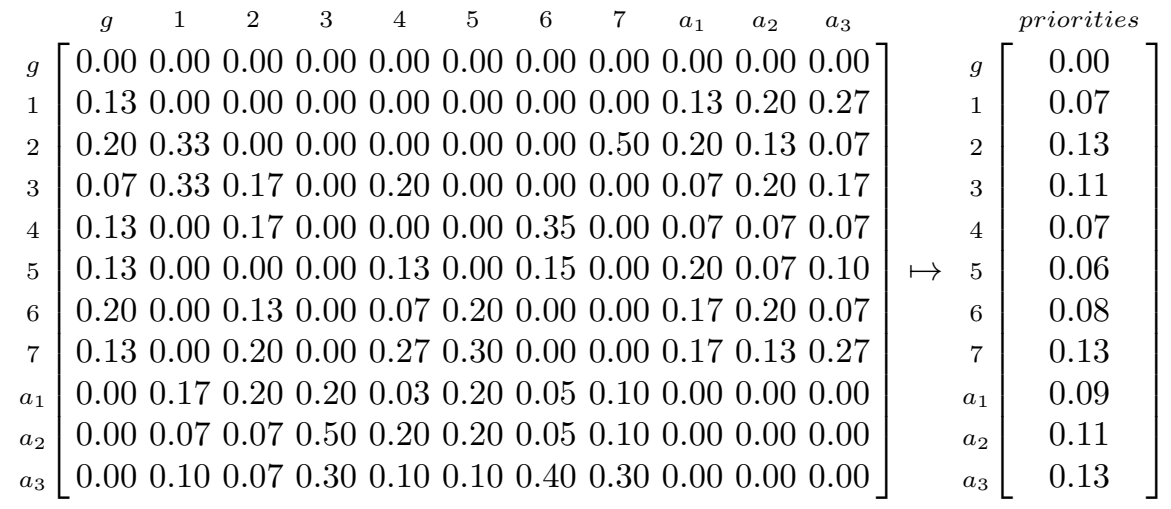

The weighted supermatrix is then transformed into a limit matrix, multiplying the weighted matrix by itself as long as all its columns become equal (Eq. 1). The values in the limit 
matrix are the final priorities of the decision-making problem. This procedure also ensures the transitivity of the connections in the decision-making problem. After achieving the final priorities, it becomes possible to conduct a sensitivity analysis and make a decision.

\section{Characteristics of the ANP}

As stated in the introduction, even though the ANP is more appropriate for strategic decision making in HE than methods which do not support modelling the influences between the criteria (like AHP), it is less often used in practice. The main focus in this research is related to identifying the characteristics of the ANP which will explain the reasons why ANP is less often used than methods which do not support modelling the influences between the criteria. These characteristics include:

1. Limitations in terms of Saaty's scale. This scale consists of only 9 degrees $[4,2]$ which sometimes seems not to be enough. However, in paper [19], the solution to this problem has been proposed.

2. "The ANP is mainly used in nearly crisp decision applications and creates and deals with a very unbalanced scale of judgment and does not take into account the uncertainty associated with the mapping of one's judgment to a number, and its ranking is rather imprecise" [1].

3. A high number of pairwise comparisons have to be done [15]. For example, in the demo example, we have to input more than 50 comparisons (which is much higher than 26 in the AHP for the same case). If the network contains a large number of dependencies between the criteria, the number of pairwise comparisons becomes higher. Generally, if $m$ is the number of clusters in the decision-making problem, $n_{i}$ is the number of elements in the $i$-th cluster and $d_{i}^{j}(k)$ is the number of dependencies of the $i$-th element from cluster $j$ considering cluster $k$, then the total number of comparisons that have to be made is $N$, respecting Eq. 2 [16].

$$
N=\sum_{k=1}^{m} \sum_{j=1}^{m} \sum_{i=1}^{n_{j}} \frac{d_{i}^{j}(k)\left(d_{i}^{j}(k)-1\right)}{2}+\frac{m^{2}(m-1)}{2}
$$

4. Conducting the inconsistency analysis is difficult without the appropriate software; therefore, when it is done by hand, there is no guarantee that it will be successful. This has an impact of the concentration of the user, and might have a negative influence on the quality of the decision-making process.

5. Influence of the decision-making problem's structure on the decision [8]; how criteria are clustered directly influences which pairwise comparisons will be made.

6. Users' misunderstandings of the pairwise comparisons of the criteria with respect to other criteria. In the demo example, criteria 6 and 7 have to be compared seven times; with respect to the goal, criteria 2, 4 and 5 and three alternatives. In the AHP, they would have to be compared only once. Users do not often differ in these comparisons; incorrect or inconsistent inputs are often given.

7. Users' misunderstandings of the pairwise comparisons of clusters with respect to other clusters. In the demo example, clusters 12 and 345 must be compared three times; with respect to the cluster $G$, the Alternatives and cluster 67 . Additional confusion comes when users must compare two clusters with respect to one of them (e.g. comparing clusters 
12 and 345 with respect to 12). Pairwise comparisons which include cluster of alternatives are even more confusing (e.g. comparing clusters 234 and the Alternatives with respect to 12). Finally, the most confusing clusters comparisons are those which include cluster of alternatives and cluster of criteria with respect to which comparison has to be made (e.g. comparing clusters 12 and the Alternatives with respect to 12).

8. The application of the property of reflexivity is not concretized in ANP theory. Does a certain criterion depend on itself? Does a certain alternative depend on itself? In Interpretative Structural Modelling (ISM), the reflexivity of the criteria is implied, and in Decision Making Trial and Evaluation Laboratory (DEMATEL), the reflexivity of the criteria is not implied. Both methods are combined with the ANP in terms of structuring. However, the ANP does not specify this detail. When discussing the alternatives, when the AHP is modelled through ANP, the reflexivity of the alternatives is mandatory [22].

High complexity is a general weakness of the ANP. Therefore, ANP implementation requires the participation of an ANP expert to be successfully applied. This process can be expensive and long, and it may lead to conflicts.

\section{Analysis of the selected ANP characteristics}

In this section, we focus on three characteristics of the ANP which have not yet been deeply studied in the literature. However, the conclusions can be very useful in ANP implementation.

\subsection{The inseparability of the criteria and alternatives}

By analysing the demo example, we can conclude that there are three elements which influence the final criteria weights: (1) comparisons of the criteria with respect to the goal, (2) comparisons of the criteria with respect to criteria (considering dependencies) and (3) comparisons which include the values of the alternatives. However, a real-case request can be that the alternatives are not known or defined at the beginning of the decision-making process; still, we need the criteria weights. A typical example for this request is the example of public procurement: before the call is published, we define the criteria and their weights, but the alternatives will be known when bidders send their offers. If we do not specify the criteria weights, leaving this to after the alternatives are defined, different frauds and irregularities are possible. As a solution for that, we can modify the ANP and calculate the criteria weights without the alternatives. Then, when the alternatives are obtained, we can calculate their priorities like in the AHP. Some other decision-making problems in which the alternatives are not known or defined can be found in $[6,10,9]$.

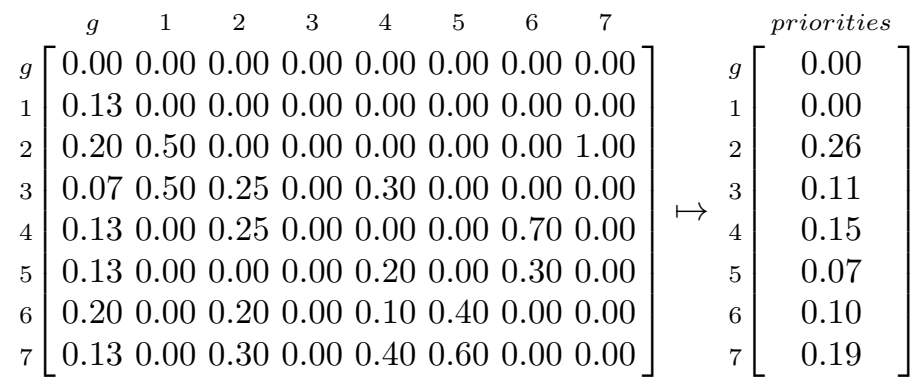

When calculating criteria weights without alternatives, the problem of irreducibility can appear. This is the case with the demo example. If we remove alternatives and calculate the limit matrix, the weight of criterion 1 becomes 0.00 (Eq. 3). This means that criterion 1 is not important for the problem. We disagree with this conclusion because, if we found that criterion 1 was 
not important, we would not put it in the network. Without the alternatives, the supermatrix is ireducibile which means that the network contains at least two criteria with no directed path between them. To solve this issue, the use of a fictive alternative $(F A)$ has been proposed $[10,9]$.

\begin{tabular}{|c|c|c|c|c|c|c|c|c|c|c|c|c|c|c|c|}
\hline & $g$ & 1 & 2 & 3 & 4 & 5 & 6 & 7 & $F A$ & & & rioritie & & & rioriti \\
\hline$g$ & 0.00 & 0.00 & 0.00 & 0.00 & 0.00 & 0.00 & 0.00 & 0.00 & 0 & & $g$ & 0.00 & & $g$ & 0.00 \\
\hline 1 & 0.13 & 0.00 & 0.00 & 0.00 & 0.00 & 0.00 & 0.00 & 0.00 & 0.14 & & 1 & 0.05 & & 1 & 0.08 \\
\hline 2 & 0.20 & 0.25 & 0.00 & 0.00 & 0.00 & 0.00 & 0.00 & 0.50 & 0.14 & & 2 & 0.12 & & 2 & 0.19 \\
\hline 3 & 0.07 & 0.25 & 0.13 & 0.00 & 0.15 & 0.00 & 0.00 & 0.00 & 0.14 & & 3 & 0.09 & & 3 & 0.15 \\
\hline 4 & 0.13 & 0.00 & 0.13 & 0.00 & 0.00 & 0.00 & 0.35 & 0.00 & 0.14 & $\mapsto$ & 4 & 0.10 & $\mapsto$ & 4 & 0.15 \\
\hline 5 & 0.13 & 0.00 & 0.00 & 0.00 & 0.10 & 0.00 & 0.15 & 0.00 & 0.14 & & 5 & 0.07 & & 5 & 0.12 \\
\hline 6 & 0.20 & 0.00 & 0.10 & 0.00 & 0.05 & 0.20 & 0.00 & 0.00 & 0.14 & & 6 & 0.08 & & 6 & 0.13 \\
\hline 7 & 0.13 & 0.00 & 0.15 & 0.00 & 0.20 & 0.30 & 0.00 & 0.00 & 0.14 & & 7 & 0.11 & & 7 & 0.18 \\
\hline$F A$ & 0 & 0.5 & 0.5 & 1.00 & 0.5 & 0.5 & 0.5 & 0.5 & 0 & & $F A$ & 0.36 & & & 1.00 \\
\hline
\end{tabular}

The fictive alternative $(F A)$ (Eq. 4)acts as a bridge which will connect any two criteria with directed path. However, the fictive alternative must not contribute to any criterion. We added a new column and a row. The priorities in the column and row are the same (except in the case of column 3, where 1 is inserted due to the stochasticity request of the ANP). Then, the limit matrix is calculated, and finally, the priorities of the criteria are normalized by their sum.

\subsection{The influence of the goal node on the priorities}

Even though the ANP allows a modelling node(s) that represent(s) one or more goals, in practice in most cases, defining the decision-making goal does not have any purpose because the priorities which resulted from the pairwise comparisons of the criteria with respect to the goal do not influence the finale criteria weights. If we change the first column of Eq. 1, the final priorities will remain the same (Eq. 5).

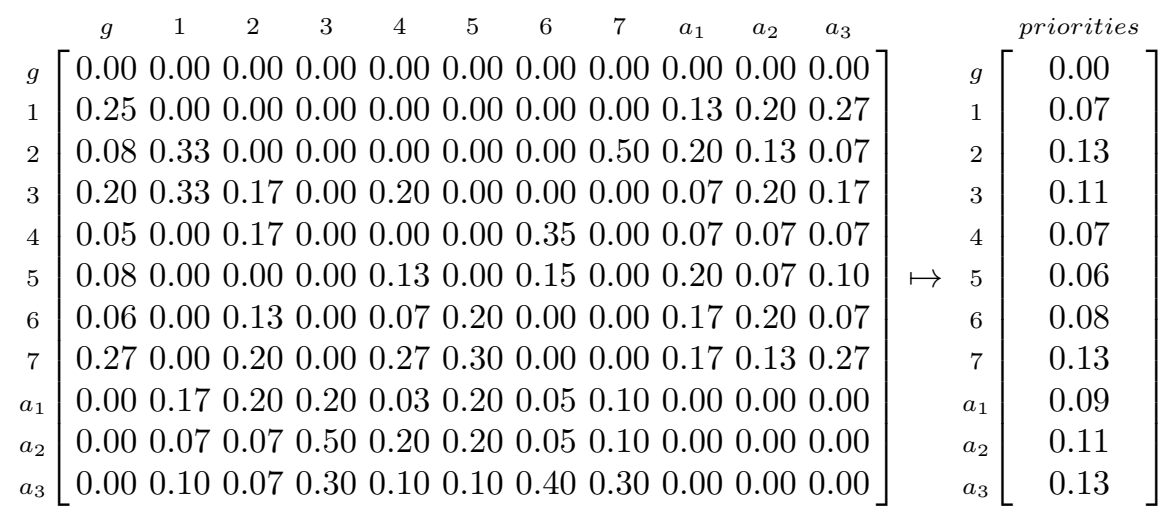

This characteristic is also related to the connectivity of the elements in the decision-making problem as well as the calculating of the limit matrix. When we include alternatives in the network (or use a fictive alternative), the network is sufficiently connected, the unweighted supermatrix converges to the limit supermatrix with all columns equal, and the priorities obtained from the pairwise comparisons of the criteria with respect to the goal do not have an influence on the final priorities.

In the case of calculating the criteria weights of a network which does not contain any alternative or a fictive alternative, when we calculate the limit supermatrix from the weighted supermatrix, there are several possible situations which can happen: 
- Unweighted supermatrix converges into limit supermatrix which contains all columns equal. The priorities obtained from the pairwise comparisons of the criteria with respect to the goal do not have an influence on the final priorities.

- Unweighted supermatrix converges into several limit matrices (rotation of the values in the limit matrices). In this case, priorities with respect to the goal have an influence on the final criteria weights. However, this situation is very rare and is almost impossible in practice. It occurs when a network contains a low number of connections which are creating one or more cycles.

- The unweighted supermatrix converges into the limit supermatrix which does not have equal columns. In this case, priorities with respect to the goal have an influence on the final criteria weights. This situation is also rare, and is almost impossible in practice. It occurs when a very low number of connections exist in the network.

- The unweighted supermatrix converges into a 0-matrix. In this case, the priorities obtained from the pairwise comparisons of criteria with respect to the goal do not have an influence on the final priorities.

The first case is the situation that occurs most often.

\subsection{The stochasticity of the supermatrix in the ANP}

The last characteristic analysed in this paper is related to the stochasticity of the supermatrix in the ANP. Regarding this characteristic, the focus is on two subcharacteristics:

- The first subcharacteristic is related to the calculation of the weighted supermatrix from the unweighted supermatrix. Besides the difficulties related to the misunderstanding of the comparisons on the cluster level, when the weighted supermatrix is calculated, clusters' priorities are adjusting to the connections within the clusters. The unweighted supermatrix of the demo case is presented in Eq. 6. To weigh the columns of the cluster 12, we must compare four clusters: 12, 345, 67 and the Alternatives. Let's say that they are equally important, which means that all clusters' weights equal 0.25 . It seems natural to multiply all values in columns 1 and 2 with 0.25 ; but if we do that, in column 1 , since there are no positive values in the rows of cluster 67 , we will not get the sum of the values in column 1 equals 1 . We will multiply the values in column 1 by $\frac{1}{3}$ because criterion 1 is connected with only three (not four) clusters. Thus, we reached the situation in which the cluster weights depended on the node structure, not the cluster structure. Similar situations will appear in the columns of clusters 345 and 67 . The calculation process has been adjusting in order to achieve stochasticity.

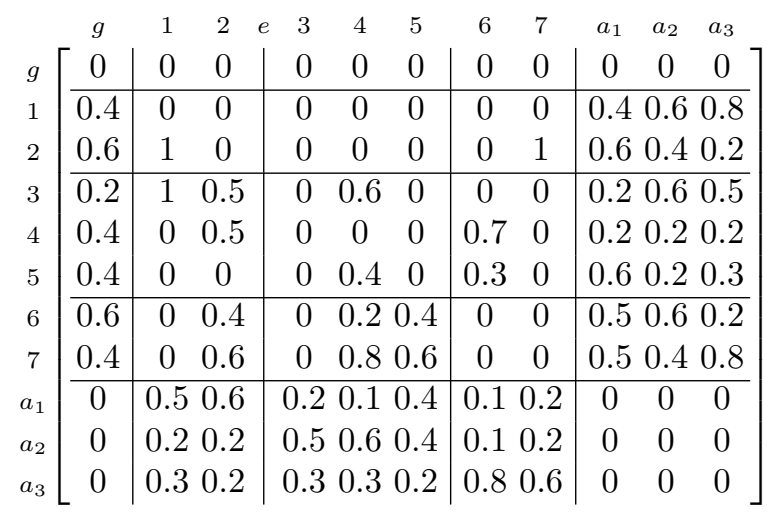


- The second subcharacteristic is related to the calculation of the priorities using the pairwise comparison procedure:

- In Eq. 6, column 2, rows 3 and 4, we compared criteria 3 and 4, determining which one of them has a greater influence on criterion 2, and how much greater. We evaluated that these influences have the same intensities, which means that they are equally important with respect to criterion 2 , and they had priorities equal to 0.5 . If the intensity of the influence of criterion 3 on criterion 2 is two times higher than the intensity of the influence of criterion 4 on criterion 2, the priorities would be 0.66 and 0.33 . From this, we can conclude that the theory of the ANP assumes that the influences between the criteria can have different intensities (low, medium, or high) and this fact has an influence on the final priorities. However, in this concrete example (comparing criteria 3 and 4 with respect to 2), we conducted the relative assessment of differences between the two influences, not their absolute values. This means that the supermatrix does not have the data about the absolute intensities of the influences, they both can be low, medium, high or very high (Figure 2). This is

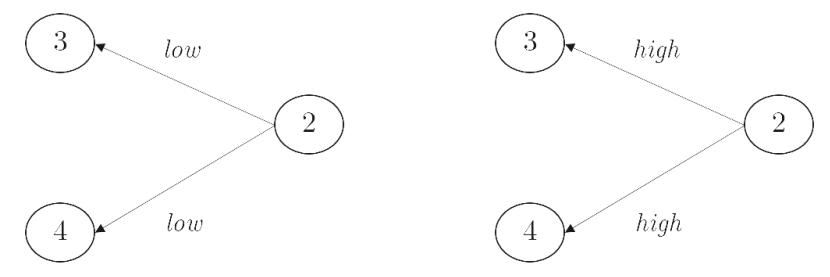

Figure 2: Two situations of equal influences between the criteria.

a weak point of the ANP method.

- In Eq. 6, column 7, row 2, the value of 1 in the supermatrix represents criterion 7 as depended on criterion 2 (or criterion 2 influences criterion 7). Also, we do not know the strength (intensity) of this dependency (influence). Logically, it seems that for final priorities, it cannot be the same if the intensity of the influence is low or high. We can conclude that the ANP will obtain the same supermatrix for the set of many different decision-making problems.

- In Eq. 6, column 7, rows 3, 4 and 5, there are three zeros because there is no influence from any of criteria 3,4 or 5 on criterion 7 . However, if we invert the paradigm and ask typical pairwise comparisons questions, we can conclude that these three criteria are equal with respect to criteria 7 . Why then, instead of zeros in the supermatrix, there is no $\frac{1}{3}$ ?

- A similar analysis can be conducted at the alternative level. If we have three alternatives which have very good values for certain criteria and are the same, the priorities will be equal to 0.33. Similarly, if we have three alternatives which have bad values but are also the same, again, the priorities will be equal to 0.33 . The good thing about the alternatives is that the criteria are mutually compared for each alternative separately (priorities will be obtained from the columns of the alternatives in the unweighted supermatrix). At this point, the supermatrix will have information that the absolute values per first criterion were good and the absolute values per second criterion were bad.

We can conclude that at the microlevel (pairwise comparisons), priorities are correct, but on the macro level (the whole supermatrix), there are some issues in the ANP steps which influence the quality of the decision making. 


\section{Conclusion}

In this paper, the ANP method was presented and demonstrated using examples. Then, the main characteristics of the ANP are listed and explained. Afterwards, a deeper analysis of three characteristics of the ANP method was conducted.

The inseparability of the criteria and alternatives in the ANP is valuable for specific contexts. For example, for any situation in which we have only the criteria level, the alternatives are not defined. There are issues with calculating the criteria weights in such situations; however, these can be decreased using the fictive alternative, and then, when the alternatives are known, applying an aggregation mechanism such as that in the AHP.

From this paper, we can conclude that no influence of priorities can be obtained through pairwise comparisons with respect to the goal of the final priorities. This information can save some time in ANP implementation (by removing the goal, we do not have to make related pairwise comparisons and get the same results).

Finally, the ANP is connected to the stochasticity property of the matrix. The stochasticity of the matrix ensures that the matrix will converge into a matrix which consists of entirely equal columns, simplifying the procedure of obtaining the finale priorities. However, this approach relativizes the decision-making problem because the matrix of the problem does not contain the absolute intensities of the influences between the criteria. Solving this issue becomes a topic for further research.

\section{Acknowledgments}

The Croatian Science Foundation supported this work under project HigherDecision IP-201409-7854.

\section{References}

[1] Ayag, Z. and Samanlioglu, F. (2016). An intelligent approach to supplier evaluation in automotive sector. Journal of Intelligent Manufacturing, 27(4), 889-903.

[2] Begičević, N. (2008). Višekriterijski modeli odlučivanja u strateškom planiranju uvođenja e-učenja. $\mathrm{PhD}$ thesis, University of Zagreb, Faculty of organization and informatics.

[3] Bhadani, A. K., Shankar, R. and Rao, D. V. (2016). Modeling the barriers of service adoption in rural Indian telecom using integrated ISM-ANP. Journal of Modelling in Management, 11(1), $2-25$.

[4] Castillo, M. and Zarama, R. (2009). Application of the analytic network process (ANP) to establish weights in order to re-accredit a program of a university. In Proceedings of the International Symposium on the Analytic Hierarchy Process, ISAHP, 1-14.

[5] Divjak, B. Development of a methodological framework for strategic decision-making in higher education - a case of open and distance learning (ODL) implementation (project application). 39.

[6] Duleba, S., Mishina, T. and Shimazaki, Y. (2012). A dynamic analysis on public bus transport's supply quality by using AHP. Transport, 27(3), 268-275.

[7] Gölcük, L. and Baykasoglu, A. (2016). An analysis of DEMATEL approaches for criteria interaction handling within ANP. Expert Systems with Applications, 46, 346-366.

[8] Hammond, J. S., Keeney, R. L. and Raiffa, H. (1998). Even swaps: a rational method for making trade-offs. Harvard business review 76, 2 .

[9] Janeš, A., Kadoić, N. and Begičević Ređep, N. (2018). Differences in prioritization of the BSC's strategic goals using AHP and ANP methods. Journal of Information and Organizational Sciences, $42(2), 1-24$.

[10] Janeš, A., Kadoić, N. and Ređep, N. B. (2017). The ANP Representation of the BSC. In CECIIS Proceedings 2017, V. Strahonja and V. Kirinić, Eds., Faculty of Organization and Informatics, 309-315. 
[11] Kadoić, N., Begičević Ređep, N. and Divjak, B. (2016). E-learning decision making: methods and methodologies. In Re-Imagining Learning Scenarios (Budapest, Hungary, 2016), vol. CONFERENCE, European Distance and E-Learning Network, p. 24.

[12] Kadoić, N., Begičević Ređep, N. and Divjak, B. (2017). A new method for strategic decisionmaking in higher education. Central European Journal of Operations Research, Special Issue of Croatian Operational Research Society and Collaborators, 26(3), 611-628.

[13] Kadoić, N., Begičević Ređep, N. and Divjak, B. (2017). Decision Making with the Analytic Network Process. In SOR 17 Proceedings (Bled, Ljubljana, 2017), M. Kljajić Borštnar, L. Zadnik Stirn, J. Žerovnik, and S. Drobne, Eds., SOR 2017, Slovenian Society Informatika, Section of Operations Research and University of Maribor, Faculty of Organizational Sciences, Slovenia Society Informatika - Section for Operational Research, 180-186.

[14] Kadoić, N., Begičević Ređep, N. and Divjak, B. (2017). Structuring e-Learning Multi-Criteria Decision Making Problems. In Proceedings of 40th Jubilee International Convention, MIPRO 2017 (Opatija, Croatia, 2017), P. Billjanović, Ed., Croatian Society for Information and Communication Technology, Electronics and Microelectronics - MIPRO, 811-817.

[15] Kadoić, N., Divjak, B. and Begičević Ređep, N. (2017). Effective Strategic Decision Making on Open and Distance Education Issues. In Diversity Matters! (Jönköping, Sweden, 2017), A. Volungeviciene and A. SzÅścs, Eds., European Distance and E-Learning Network, 224-234.

[16] Kadoić, N., Divjak, B. and Begičević Ređep, N. (2017). Integrating the DEMATEL with the Analytic Network Process for Effective Decision-Making. Central European Journal of Operations Research, S.I. of SOR 2017 conference (unpublished), 1-30.

[17] Saaty, T. L. (1999). Fundamentals of the Analytic network Process. Japan, Kobe: The International Symposium on the Analytic Hierarchy Process.

[18] Saaty, T. L. (2001). Decision Making with Dependence and Feedback: The Analytic Network Process : the Organization and Prioritization of Complexity, second ed. RWS Publications, New York.

[19] Saaty, T. L. (2006). The Analytic Network Process âĂŞ Dependence and Feedback in DecisionMaking: Theory and Validation Examples. In Business Applications and Computational Intelligence, K. Voges and N. Pope, Eds. IGI Global, 360-388.

[20] Saaty, T. L. (2008). Decision making with the analytic hierarchy process. Int. J. Services Sciences, $1(1), 83-98$.

[21] Saaty, T. L. and Cillo, B. (2008). A Dictionary of Complex Decision Using the Analytic Network Process, The Encyclicon, Volume 2, 2nd ed. RWS Publications, Pittsburgh.

[22] Saaty, T. L., and Vargas, L. G. (2006). Decision Making with the Analytic Network Process: Economic, Political, Social and Technological Applications with Benefits, Opportunities, Costs and Risks. Springer; Softcover reprint of hardcover 1st ed. 2006 edition (December 28, 2009).

[23] Saaty, T. L. and Vargas, L. G. (2013). The Analytic Network Process. In Iranian journal of operational research, 1, 1-28.

[24] Thakkar, J., Deshmukh, S. G., Gupta, A. D. and Shankar, R. (2005). Selection of Third-Party Logistics (3PL): A Hybrid Approach Using Interpretive Structural Modeling (ISM) and Analytic Network Process (ANP). Supply Chain Forum: an International Journal, 6(1), 32-46.

[25] Tsai, W.-H. and Hsu, W. (2010). A novel hybrid model based on DEMATEL and ANP for selecting cost of quality model development. Total Quality Management \& Business Excellence, 21(4), 439-456.

[26] Yang, J. L., and Tzeng, G. H. (2011). An integrated MCDM technique combined with DEMATEL for a novel cluster-weighted with ANP method. Expert Systems with Applications, 38(3), 14171424.

[27] Yang, Y., Shieh, H., Leu, J. and Tzeng, G.-H. (2008). A novel hybrid MCDM model combined with DEMATEL and ANP with applications. International Journal of Operations Research, 5(3), 160-168. 